Schutz vor einer Verschiebung des gesellschaftlichen Kräfteverhältnisses zugunsten einflussreicher und wirtschaftlich starker gesellschaftlicher Gruppen.

Während die Prüfung der verfassungsrechtlichen Bedingungen und Grenzen von Privatisierung umfassend erfolgt, reißt der Autor die gesellschafts- und staatstheoretischen Aspekte in den Beispielfeldern oft eher an als dass er sie komplett ausführt. Es ist spürbar, wie komplex und raumgreifend eine (auch) marxistisch-orientierte Analyse hier sein kann. Da es zudem in der Rechtswissenschaft $\mathrm{zu}$ marxistisch orientierten Ansätzen keinen allseits bekannten Wissensbestand gibt, muss der Autor zudem zunächst sehr grundsätzlich an die Beschreibung gehen.

Insgesamt ist Julian Zado damit ein sehr interessanter Beitrag zur Debatte über Sinnhaftigkeit und Grenzen von Privatisierungen gelungen. Besonders hervorzuheben ist, dass der Autor nicht schlicht „Staatsaufgaben“ "beschreibt, sondern der Funktion von Staatlichkeit in entwickelten industriell-kapitalistischen Gesellschaften nachspürt. Damit gelingt es ihm, die Verbundenheit von Gesellschaftsanalyse und rechtspolitischer Debatte deutlich zu machen. Es wäre sehr zu wünschen, dass dieser methodische Mut Nachahmer findet.

Thilo Scholle

Matthias Lehnert, Frontex und operative

Maßnabmen an den europäischen Außengrenzen:

Verwaltungskooperation - materielle

Rechtsgrundlagen - institutionelle Kontrolle,

Baden-Baden (Nomos-Verlag [Schriften zum

Migrationsrecht, Band 12]) 2014, 579 S., $€ 139,00$

In seiner 2014 erschienenen Dissertation widmet sich Matthias Lehnert einem juristisch sowie politisch kontrovers diskutierten Thema: dem Schutz der europäischen Außengrenzen, mit anderen Worten dem Schutz des Raumes der Freiheit, der Sicherheit und des Rechts. Während die EU ihren Bürger_innen „einen Raum der Freiheit, der Sicherheit und des Rechts ohne Binnengrenzen“ gewährleisten will, konstatiert sie zugleich eine Politik der Kontrolle an den Außengrenzen, des Asyls und der Einwanderung (Art. 3 EUV, Art. 77 AEUV). Und in der Tat führte die europäische Integration zeitgleich zur Abschaffung der europäischen Binnengrenzen und Schaf- fung einer gemeinsamen europäischen Außengrenze. Der Schutz dieser europäischen Außengrenze ist nunmehr nicht nur die Aufgabe der einzelnen Staaten, sondern er ist $\mathrm{zu}$ einem europäischen Interesse avanciert.

Um dieses gemeinsame Interesse zu verfolgen, wurde 2004 die Europäische Grenzschutzagentur Frontex gegründet. In dieser Agentur treffen zahlreiche parallel verlaufende Prozesse der Europäischen Union zusammen. Zum einen sind das Politiken rund um die Migration, insbesondere: Europäisierung der Grenzen, Externalisierung der Migrationskontrolle und zunehmende Illegalisierung der Migration und der Migrant_innen. Das sind Entwicklungslinien, die sich zugleich mit der Verwendung von Vokabularien wie Migrationsströme und Bekämpfung von Kriminalität gut eignen, um die Migration in einer Sicherheitslogik zu bearbeiten und eine Existenzbedrohung zu suggerieren, die dazu verhelfen soll, (menschen-)rechtliche Maßstäbe und Vorgaben zu relativieren, sich ihnen zum Teil sogar zu entledigen. Zum anderen handelt es sich um grundsätzliche Prozesse in der EU: zunehmende Verwaltungskooperation (Mehrebenenverwaltung) und die Auslagerung der Aufgaben in Subinstitutionen (Agenturen) der EU. Dieser zuletzt genannte Komplex ist aus mehrfacher Sicht spannend: Sei es die Frage nach der rechtlichen Kontrolle der Handlungen einzelner Akteure oder nach der Legitimation (Demokratiedefizit) der Institutionen - die angrenzenden Themen sind in der öffentlichen Diskussion sehr präsent.

Vor diesem Hintergrund will Lebnert, an die operativen Maßnahmen an den Außengrenzen anschließend, die Tätigkeit von Frontex aus einer rechtlichen Perspektive beleuchten. Als Untersuchungsmaßstab zieht der Verfasser grundlegend das Unionsrecht heran. Daneben werden die Genfer Flüchtlingskonvention (GFK), die Europäische Menschenrechtskonvention (EMRK) und das Seevölkerrecht in die Prüfung einbezogen.

Die Arbeit gliedert sich in fünf Teile: Der erste Teil widmet sich der Europäischen Grenzschutzagentur (Kapitel 1 und 2). Im zweiten Teil wird die Untersuchung auf Verwaltungskooperation im Aktionsradius von Frontex zugeschnitten (Kapitel 3 und 4). Dem schließt sich im dritten Teil die Frage nach dem materiellen Rechtsrahmen operativer Maßnahmen und ihrer RechtmäBigkeit an (Kapitel 5-7). In den letzten zwei Tei- 
len wird zum einen der individuelle gerichtliche Rechtsschutz bei operativen Maßnahmen an den Außengrenzen untersucht (Kapitel 8-13) und zum anderen die außergerichtliche Kontrolle der operativen Maßnahmen und des institutionellen Rahmens der Agentur unter die Lupe genommen (Kapitel 14 und 15).

Im ersten Teil gibt der Verfasser einen umfassenden Überblick. Neben der Erörterung der rechtlichen Grundlagen wird der organisatorische Rahmen veranschaulicht. Insbesondere wird die interne Organisation und die Kooperation mit Mitgliedstaaten, mit sog. Drittstaaten sowie mit Organisationen wie z. B. UNHCR und IOM, dargestellt (S. 47-75). Mit der Darstellung der vielfältigen Aufgaben von Frontex, wobei der Fokus auf den operativen Maßnahmen an den Außengrenzen liegt, wird der erste Teil abgeschlossen. Dabei wird präzise die Beteiligung und Mitwirkung der einzelnen Akteure an den Maßnahmen herausgearbeitet. Dieser Teil ist nicht nur sehr informativ, sondern es wird gut ersichtlich, dass die Agentur nicht isoliert als eine Unionsbehörde handelt, sondern auf die Mitgliedstaaten als Kooperationspartner zurückgreift. Bei den operativen Maßnahmen wird eine „neuartige, delokalisierte Form des Grenzmanagements" praktiziert, so der Verfasser (S. 127). Ebenso gelingt es zu zeigen, dass Frontex faktisch einen maßgeblichen Einfluss auf die Ausgestaltung und Durchführung der Maßnahmen hat.

Der Verfasser setzt sich im Anschluss ausführlich mit der Verwaltungskooperation im Unionsrecht im Allgemeinen und in Bezug auf Frontex auseinander. Lebnert gelingt es sehr gut, die komplexe Verwaltungskooperation in seinen unterschiedlichen Formen und Ebenen näher zu bringen. Die Kooperationsformen, an denen Frontex beteiligt ist, werden sodann einer dogmatischen Einordnung und Kontextualisierung unterzogen (S. $160 \mathrm{ff}$.). Sorgfältig werden die einzelnen Kooperationen differenziert und analysiert. Diese Differenzierung bedeutet eine wichtige Weichenstellung für Fragen des Rechtsschutzes. Kritisch stellt Lebnert fest, dass indem „nunmehr nicht nur eine Institutionalisierung, sondern zugleich eine Koordinierungskompetenz auf die unionale Ebene verlagert wurde, die in der Praxis von weitreichender Bedeutung ist, [...] Frontex in diesem Bereich für eine teilweise Hochzonung von Kompetenzen [steht]. “ (S. 164).
Eine Fülle von Problemen und Fragen werden im dritten Teil im Rahmen einer sorgfältigen Analyse der operativen Maßnahmen von Frontex behandelt (S. $171 \mathrm{ff}$.). Sei es die Tatsache, dass bei den Maßnahmen mehrere Hoheitsträger beteiligt sind, die diversen Lokalitäten, an denen die Operationen stattfinden, oder der die rechtliche $\mathrm{Be}-$ wertung verkomplizierende Umstand, dass die Maßnahmen schwerpunktmäßig auf See stattfinden, sodass die besondere Berücksichtigung des Seevölkerrechts erforderlich ist, erörtert der Verfasser kritisch.

Die einschlägigen Normen aus der GFK, EMRK und Grundrechtecharta (GRC) werden sowohl im Falle der territorialen als auch der extraterritorialen Handlungen erörtert. Die extraterritoriale Geltung der jeweiligen Vertragswerke und Normen wird nach einer knappen Erörterung in Anlehnung an die Jurisdiktionsausübung festgestellt. Sodann werden die inhaltlichen Vorgaben aus der GFK, EMRK und GRC sowie aus Seevölkerrecht präzise und ausführlich bearbeitet, bevor der Frage nachgegangen wird, ob entsprechend dem Grundsatz des Vorbehalts des Gesetzes die gesetzlichen Grundlagen für die operativen Maßnahmen der Grenzschutzagentur vorliegen (S. $249 \mathrm{ff}$.). Nach einer umfassenden Analyse hält Lehnert als Ergebnis im Wesentlichen fest, dass die Grenzschutzmaßnahmen unter Koordinierung von Frontex weitreichenden Beschränkungen, insbesondere non-refoulementGeboten, aus der GFK, EMRK und GRC unterliegen. Die inhaltlichen Vorgaben richten sich auf die Gewährleistung eines effektiven und individuellen Verfahrens für schutzsuchende Personen, welche regelmäßig das Recht auf Zugang zum Staatsgebiet nach sich zieht - da faktisch die Gewährleistung der Rechte aus den genannten Vertragswerken sonst nicht möglich ist. Der Verfasser erörtert darüber hinaus die Maßnahmen auch in Bezug auf das Seevölkerrecht. Hierbei stellt er fest, dass die Durchführung von Grenzschutzmaßnahmen im mitgliedstaatlichen Küstenmeer, der Anschlusszone, auf Hoher See und auf dem seewärtigen Gebiet von Drittstaaten verboten sind (S. 272). Zugleich wird eine Verpflichtung der Staaten zur Seenotlage und ein Nothafenrecht aus dem Seevölkerrecht festgestellt. In Anlehnung an diese Feststellungen dürften sich die meisten operativen Grenzschutzmaßnahmen von Frontex als völkerrechtswidrig erweisen. Auch das einschlägige unionale Sekundärrecht ent- 
spricht nur begrenzt den menschenrechtlichen Vorgaben.

Die Arbeit verdeutlicht in diesem Teil mit fundierten und überzeugenden Ausführungen, was die Gewährung eines Raumes „der Freiheit, der Sicherheit und des Rechts ohne Binnengrenzen" für die Bürger_innen der EU für alle anderen außerhalb dieses Raumes praktisch bedeutet: Der Raum rund um die Festung Europa wird im Grunde zu einem Raum, in dem sich die EU und ihre Mitgliedstaaten dem Recht zu entledigen suchen. Ein effektives individuelles Verfahren zur Feststellung der Flüchtlingseigenschaft wird nicht bereitgestellt, das refoulement-Verbot wird untergraben, das Verbot der Kollektivausweisung und der von Rechts wegen erforderliche Zugang zum Staatsgebiet wird kaum beachtet (vgl. zusammenfassend S. 500).

Die letzten beiden Teile der Arbeit widmen sich Aspekten der gerichtlichen und außergerichtlichen Kontrolle der operativen Maßnahmen und der Agentur. Auch hier ist die Untersuchung breit angelegt und die einzelnen Aspekte sorgfältig differenziert. Neben der Erörterung der Handlungen der Beamt_innen des Einsatzmitgliedstaates und der anderen Mitgliedstaaten setzt sich Lehnert ausführlich mit dem Rechtsschutz gegenüber Frontex auseinander (S. 333-416). Dabei zeigt der Verfasser, warum ein Primärrechtsschutz gegen Frontex ausgeschlossen ist, welche Herausforderungen beim Sekundärschutz bestehen und wie sie zu lösen sind. An die Zurechnungsfigur der „effektiven Kontrolle“ anschließend, welche er für die Maßnahmen der Frontex an den Außengrenzen fruchtbar macht (S. $341 \mathrm{ff}$.), exploriert der Verfasser vielschichtige Möglichkeiten des Rechtschutzes gegen Frontex (S. 397 ff.).

Der letzte Teil der Arbeit analysiert die außergerichtliche Kontrolle des institutionellen Rahmens und der Handlungen der Agentur. Auch wenn Lehnert engagiert nach außergerichtlichen Möglichkeiten der Kontrolle sucht, ist das Ergebnis ernüchternd. Denn die Effektivität der auBergerichtlichen Kontroll- und Beschwerdemöglichkeiten ist aus unterschiedlichen Gründen wenig Erfolg versprechend.

Jenseits der einzelnen Abschnitte, deren gestraffte Darstellung der Arbeit besser getan hätte, handelt es sich insgesamt um eine sehr verdienstvolle Abhandlung. Die Arbeit liest sich sehr gut, sie ist sehr informativ und bietet eine Fülle von weiterführenden Ergebnissen, die der Verfasser durchweg argumentativ präzise und fundiert begründet. Aus der Arbeit sind nicht nur analytische, sondern auch kritische rechtspolitische Impulse zu entnehmen. Wer sich umfassend über den Grenzschutz, die Grenzschutzagentur, ihre Maßnahmen und die rechtliche Bewertung der einzelnen Themenkomplexe informieren will, oder wer sich nur für einzelne Aspekte interessiert, dem kann die Lektüre des Buches sehr empfohlen werden.

\section{Ibrabim Kanalan}

\section{Hans Jörg Sandkühler, Recht und Staat nach menschlichem Maß. Einführung in die Rechts- und Staatstheorie in menschenrechtlicher Perspektive. Weilerswist (Velbrü̈k Wissenschaft) 2013; 688 S., $€ 49,90$}

Hans Jörg Sandkühler - von 1974 bis 2005 Professor für Philosophie an der Universität Bremen und von 2003 bis 2011 Leiter der Deutschen Abteilung „Menschenrechte und Kulturen“ des europäischen UNESCO-Lehrstuhls für Philosophie in Paris - legt mit seinem umfangreichen Werk eine Einführung in die allgemeine Rechtsund Staatstheorie vor, der es ,um eine von der Rechtsnorm der Achtung und des Schutzes der Menschenwürde ausgehende und durch die positivierten Menschenrechte begründete pluralistische, Rechtspluralismus anerkennende und zugleich nicht durch Kultur- und Rechtsrelativismus unterhöhlte normative Theorie von Recht, Staat und Demokratie“ (11) geht.

Sandkühler nimmt die Ausdifferenzierung des Rechtssystems ernst, indem er betont, dass die normativen Grundlagen des Rechts, aber auch der Rechts- und Staatskritik, aus dem Rechtssystem selbst gewonnen werden müssen und nicht (länger) aus der notwendig pluralistisch, ja relativistisch verfassten moralischen Kommunikation importiert werden können $(12,220 \mathrm{ff}$.). Er geht davon aus, dass die Maßstäbe für die Entwicklung und die Kritik von Recht und Staat heute in der Menschenwürdenorm und den Normen des internationalen Menschenrechte-Regimes - der „einzige[n] Universalität, die wir in dieser polymorphen Welt mit tatsächlicher Geltung haben können“ (52) - vorliegen. Das Buch buchstabiert diese "Grundlegungsfunktion“ (12) der Menschenwürde und der Menschenrechte für das nationale und internationale Recht, für den Natio- 